\title{
CONNECTIVITY AND EDGE-BIPANCYCLICITY OF HAMMING SHELL.
}

\author{
S. A. MANE AND B. N. WAPHARE \\ Center for Advanced Studies in Mathematics, Department of Mathematics, \\ Savitribai Phule Pune University, Pune-411007, India. \\ manesmruti@yahoo.com : waphare@yahoo.com
}

\begin{abstract}
An Any graph obtained by deleting a Hamming code of length $n=2^{r}-1$ from a $n$-cube $Q_{n}$ is called as a Hamming shell. It is well known that a Hamming shell is vertex-transitive, edge-transitive, distance preserving [1 2, 3, 4. Moreover, it is Hamiltonian [6] and connected [5] . In this paper, we prove that a Hamming shell is edge-bipancyclic and $(n-1)$-connected.
\end{abstract}

Keywords : Perfect independent domination(perfect code), distant faulty vertices, connectivity, edge-bipancyclicity, Hypercubes.

\section{INTRODUCTION}

The hypercube $Q_{n}$ of dimension $n$ is one of the most versatile and powerful interconnection networks. The graph of $Q_{n}$ has many excellent properties such as recursive structure, regularity, symmetry, small diameter, low degree etc.

A binary linear code of length $n=2^{r}-1(r \geq 2)$, with parity-check matrix $H$ whose columns consist of all the nonzero vectors of $F_{2}^{r}$ is called a binary Hamming code of length $2^{r}-1$. Binary Hamming codes are perfect codes (perfect independent dominating sets). Any graph obtained by deleting a Hamming code of length $n=2^{r}-1$ from a $n$-cube $Q_{n}$ is called as a Hamming shell.

The study of the effect of removal of a dominating set on a network is interesting in the sense that the damage that the network experiences is more when a core of the network is removed. In interconnection networks, it means that when a dominating set is removed, the network suffers a heavy set back when the remaining part of the network gets scattered. This gives the motivation to study the vulnerability of a network when dominating sets are removed. For example, in a network with a node having connections with all other nodes which are independent, the removal of this master node will result in complete chaos. On the other hand, when every node has connection with every other node in a network, then the removal of any number of vertices will not affect the cohesiveness of the remaining part. But, such a network is costly. We have to examine various networks which are economic and at the same time have a high ability to withstand attacks on dominating sets.

Connectivity is often used as a measure of system reliability and fault tolerance.

Cycles the most fundamental classes of network topologies for parallel and distributed computing. Cycles are suitable for designing simple algorithms with low communication costs. Numerous efficient parallel algorithms designed on cycles for solving various algebraic problems, graph problems, and some parallel applications, such as those in image and signal processing. These algorithms can also be used as control/data flow structures for distributed computing in arbitrary networks if these networks can embed cycles so that the algorithms designed on cycles can be executed on the embedded cycles. In addition, if cycles of various lengths can be embedded, the number of simulated processors can be adjusted to increase the elasticity of demand. As a result, the problem of embedding cycles of various lengths into a topology is crucial for network simulation. In this paper, we tried to measure the fault tolerant bipancyclicity and connectivity of a hypercube when its dominating sets are under attack. 
The Dejter graph is obtained by deleting a copy of the Hamming code of length 7 from the binary 7 -cube. The Dejter graph, and by extension any graph obtained by deleting a Hamming code of length $2^{r}-1$ from a $\left(2^{r}-1\right)$-cube is well known Hamming Shell which is vertex-transitive, edgetransitive, distance preserving [1, 2, 3, 4, Also, Gregor and Skrekovski [6] proved that Hamming Shell is Hamiltonian. Moreover, Duckworth et al. [5] proved a lemma, that Hamming Shell is connected. Thus, a Hamming shell preserves many useful properties of hypercube.

Inspiring from above applications and results, in this paper, we prove that a Hamming shell preserves two more useful properties of hypercube those are edge-bipancyclicity and $(n-1)$-connectivity.

Weichsel[11] considered perfect dominating sets in hypercubes as a way of generating distance preserving regular subgraphs. In this paper, we prove the existence of perfect dominating set $D$ of $Q_{n}(n \geq 3)$ such that $Q_{n}-D$ is $(n-1)$-regular, $(n-1)$-connected and bipancyclic.

For undefined terminology and notations see West [12] and Ling and Xing $[8]$.

\section{PRELiminaries}

An $n$-dimensional hypercube $Q_{n}$ can be represented as an undirected graph $Q_{n}=(V, E)$ such that $V$ consists of $2^{n}$ nodes which are labeled as binary numbers of length $n$. $E$ is the set of edges that connect two nodes if and only if they differ in exactly one bit of their labels. The parity of a vertex in $Q_{n}$ is the parity of the number of $1 \mathrm{~s}$ in its name, even or odd. As a number of even parity vertices is same as a number of odd parity vertices in $Q_{n}$ and hence it is balanced. $Q_{n}$ has many attractive properties, such as being bipartite, $n$-regular, $n$-connected. It is a bipancyclic graph in the sense that, for every even integer k, $4 \leq k \leq V\left(Q_{n}\right)$, there exists a cycle $C_{k}$ of length $k$ in $Q_{n}$. Moreover, it is an edge-bipancyclic graph, as for any edge in $Q_{n}$, there is a cycle $C_{k}$ of every even length $k, 4 \leq k \leq V\left(Q_{n}\right)$, traversing through this edge.

$Q_{n+1}$ can be decomposed into two copies of $Q_{n}$ (denoted by $\left(Q_{n}, 0\right)$ and $\left.\left(Q_{n}, 1\right)\right)$ whose vertices are joined by $2^{n}$ edges of a perfect matching $R$. These edges are called cross edges. Let $V\left(Q_{n}\right)=$ $\left\{v_{i}: 1 \leq i \leq 2^{n}\right\}$ and without $\operatorname{loss} V\left(Q_{n+1}\right)=\left\{\left(v_{i}, 0\right),\left(v_{i}, 1\right): v_{i} \in V\left(Q_{n}\right), 1 \leq i \leq 2^{n}\right\}$. So, we write $Q_{n+1}=\left(Q_{n}, 0\right) \cup\left(Q_{n}, 1\right) \cup R$ where $V\left(Q_{n}, 0\right)=\left\{\left(v_{i}, 0\right): v_{i} \in V\left(Q_{n}\right), 1 \leq i \leq 2^{n}\right\}$, $V\left(Q_{n}, 1\right)=\left\{\left(v_{i}, 1\right): v_{i} \in V\left(Q_{n}\right), 1 \leq i \leq 2^{n}\right\}$. Note that the end vertices of any edge in $R$ are called corresponding vertices of each other.

For $n=2^{m}-1$ and $m \geq 2$ if we denote by $k=2^{m+1}-1$ obviously $k=n+(n+1)$. We decompose $Q_{k}=Q_{n} \times Q_{n+1}$. Now for any $t \in V\left(Q_{n+1}\right)$, we denote by $\left(Q_{n}, t\right)$ the subgraph of $Q_{k}$ induced by the vertices whose last $n+1$ components form the tuple $t$ and $(D, t)$ denotes the subgraph of $\left(Q_{n}, t\right)$. It is easy to observe that $\left(Q_{n}, t\right)$ is isomorphic to $Q_{n}$.

$F \subset V\left(Q_{n}\right)$ is said to be distant (strongly independent) set of vertices if $N[u] \cap N[v]=\phi$ for every $u, v \in F$.

\section{3. construction of a perfect independent dominating (PID) set of $Q_{2^{m+1}-1}$ from PID set of $Q_{2^{m}-1}, m \geq 2$.}

In this section, we prove a lemma which provides a technique of constructing a PID set of $Q_{2^{m+1}-1}$ from PID set of $Q_{2^{m}-1}, m \geq 2$. We consider $Q_{n}$ as a vector space over $Z_{2}$.

Whenever $n$ is of the form $n=2^{m}-1(m \geq 2)$, there exists a strongly independent subset of $Q_{n}$ which is a dominating set and hence a perfect independent dominating set. In fact, if $H$ is an $m \times\left(2^{m}-1\right)$-matrix obtained by taking all non-zero $m$-tuples of $Q_{m}$ as columns of $H$ then the kernel of the linear transformation $H: Q_{n} \longrightarrow Q_{m}$ is a perfect independent dominating set of $Q_{n}$ (see [7]). It is clear that any linear subspace which is a perfect independent dominating set is a kernel of such type of matrix (see [4]). 
Lemma 3.1. If $D^{\prime}$ is PID linear subspace of $Q_{n}\left(n=2^{m}-1\right)$ then $D$ is PID set which is also a linear subspace of $Q_{n+n+1}$ where $D=\left\{(x, y, j): x \in V\left(Q_{n}\right), y \in\left(D^{\prime}+x\right), \sum y_{i}=j\right\}$

Proof. Let

$$
\left[\begin{array}{ccc}
H^{\prime} & H^{\prime} & 0 \\
0 & 1 & 1
\end{array}\right]
$$

Now $H(x, y, j)^{T}=0$

iff $\sum y_{i}=j$ and $H^{\prime} x+H^{\prime} y=0$.

iff $\sum y_{i}=j$ and $x+y \in D^{\prime}$.

iff $\sum y_{i}+j=0$ and $x \in V\left(Q_{n}\right)$ and $y \in\left(D^{\prime}+x\right)$. Thus for any $x \in V\left(Q_{n}\right)$ choose $y \in\left(D^{\prime}+x\right)$ and choose $j=\sum y_{i}$ to form $D$.

Observation 3.2. For $m \geq 2$ denote by $n=2^{m}-1$ and $k=2^{m+1}-1=n+n+1$. Let $Q_{k}=Q_{n} \times Q_{n+1}$. By Lemma 3.1, It is easy to observe that

[a] for any linear PID set $D_{0}^{\prime}$ of $Q_{n}, D_{i}^{\prime}=D_{0}^{\prime}+e_{i}$ is also a PID set for every $i(1 \leq i \leq n)$ and further $V\left(Q_{n}\right)=\bigcup_{i=0}^{n} D_{i}^{\prime}, D_{i}^{\prime} \cap D_{j}^{\prime}=\phi$ for $i \neq j(0 \leq i, j \leq n)$. For every $i(0 \leq i \leq n), D_{i}^{\prime}$ is balanced

[b] for odd parity vertex $t \in V\left(Q_{n+1}\right), D \bigcap V\left(Q_{n}, t\right)=\phi$ and for even parity vertex $t \in V\left(Q_{n+1}\right)$, $D \cap V\left(Q_{n}, t\right)=\left(D_{i}^{\prime}, t\right)(0 \leq i \leq n)$.

\section{Edge-bipancyclicity of hypercubes with faulty vertices in PID set.}

To prove our main result of this section we need following lemmas.

Lemma 4.1 ([13]). Any two edges in $Q_{n}(n \geq 2)$ are included in a Hamiltonian cycle.

Lemma 4.2. For any PID set $D$ of $Q_{3}, Q_{3}-D$ is Hamiltonian.

Proof. see Figure 1.
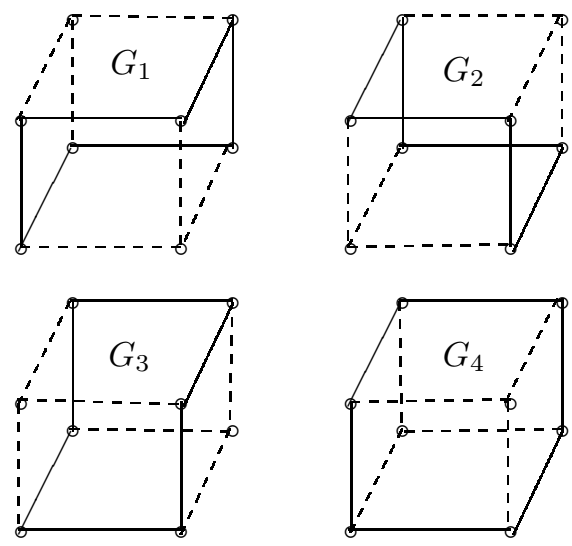

Figure 1

Lemma 4.3. Consider the subgraphs $\left(Q_{3}, 0\right)$ and $\left(Q_{3}, 1\right)$ of $Q_{4}$. Let $G^{\prime}$ be a cycle of $\left(Q_{3}, 0\right)$ with $\left|V\left(G^{\prime}\right)\right|=6$ and $R^{\prime}=$ set of cross edges between $\left(Q_{3}, 1\right)$ and $G^{\prime}$. Consider $G=\left(Q_{3}, 1\right) \cup R^{\prime} \cup G^{\prime}$. Then $G$ is an edge-bipancyclic graph. 
Proof. It is easy to observe that any two cycles of length greater equal 6 in $Q_{3}$ have at least 4 vertices common, and since a degree of each vertex is 3 so at least two edges common.

For every edge $T \in E(G)$, we want a cycle of every even length $l(4 \leq l \leq|V(G)|)$ passing through $T$. (see Figure 2)

For edges $e^{\prime}, f^{\prime}, h^{\prime} \in E\left(G^{\prime}\right)$ let us denote their corresponding edges in $E\left(Q_{3}, 1\right)$ by $e, f, h$ respectively. Also, let us denote $e_{1}, e_{2}, e_{3}, e_{4} \in R^{\prime}$ the edges out of which $e_{1}, e_{2}$ join the end vertices of $e$ and $e^{\prime}, f_{1}, f_{2}$ join the end vertices of $f$ and $f^{\prime}$ and $h_{1}, h_{2}$ join the end vertices of $h$ and $h^{\prime}$

Case 1: For an edge $e$ in $E\left(Q_{3}, 1\right)$. As $\left(Q_{3}, 1\right)$ is an edge-bipancyclic graph therefore there exists a cycle of every even length $l\left(4 \leq l \leq\left|V\left(Q_{3}\right)\right|\right)$ in $\left(Q_{3}, 1\right)$ hence in $G$ containing $e$. Let $C$ be a Hamiltonian cycle in $\left(Q_{3}, 1\right)$ containing an edge $e$. Now, other than $e$ there is at least one edge say $f$ lies on cycle $C$ such that its corresponding edge $f^{\prime}$ is on cycle $G^{\prime}$. Consider cycles $(C-f)+f_{1}+f_{2}+f^{\prime}$ and $(C-f)+f_{1}+f_{2}+\left(G^{\prime}-f^{\prime}\right)$ which contain $e$ with their length 10 and 14 respectively. Lastly, let $C_{6}$ be a cycle of length 6 in $\left(Q_{3}, 1\right)$ which contains an edge $e$ and let $h$ be any other edge on this cycle which have its corresponding edge say $h^{\prime}$ in $G^{\prime}$. Now we construct a cycle of length 12 containing $e$ as $\left(C_{6}-h\right)+h_{1}+h_{2}+\left(G^{\prime}-h^{\prime}\right)$.

Case 2: Consider an edge $e^{\prime}$ in $E\left(G^{\prime}\right)$, let $f^{\prime} \neq e^{\prime}$ be an edge on $G^{\prime}$ ( there are 5 such edges). Consider $C_{k}$ be a cycle in $\left(Q_{3}, 1\right)$ of even length $k\left(4 \leq k \leq\left|V\left(Q_{3}\right)\right|\right.$ containing $f$. Now we construct a cycle containing $e^{\prime}$ of even length $l=k+\left|V\left(G^{\prime}\right)\right|$ (as $4 \leq k \leq\left|V\left(Q_{3}\right)\right|$ therefore $\left.\left|V\left(G^{\prime}\right)\right|+4 \leq l \leq\left|V\left(G^{\prime}\right)\right|+\left|V\left(Q_{3}\right)\right|\right)$ as $\left(G^{\prime}-f^{\prime}\right)+f_{1}+\left(C_{k}-f\right)+f_{2}$. For $l=\left|V\left(G^{\prime}\right)\right|+2$ consider a cycle $\left(G^{\prime}-f^{\prime}\right)+f_{1}+f+f_{2}$ and for $l=4$ a cycle is $e^{\prime}+e_{1}+e_{2}+e$ containing $e^{\prime}$.

Case 3: For an edge $f_{1}$ in $R^{\prime}$. let one end vertex of $f_{1}$ is common with an edge $f^{\prime}$ in $G^{\prime}$ then Consider $C_{k}$ be a cycle in $\left(Q_{3}, 1\right)$ of even length $k\left(4 \leq k \leq\left|V\left(Q_{3}\right)\right|\right.$ containing $f$. Now we construct a cycle containing $f_{1}$ of even length $l=k+\left|V\left(G^{\prime}\right)\right|$ ( therefore $\left|V\left(G^{\prime}\right)\right|+4 \leq l \leq\left|V\left(G^{\prime}\right)\right|+\left|V\left(Q_{3}\right)\right|$ ) as $\left(G^{\prime}-f^{\prime}\right)+f_{1}+\left(C_{k}-f\right)+f_{2}$. For $l=\left|V\left(G^{\prime}\right)\right|+2$ consider a cycle $\left(G^{\prime}-f^{\prime}\right)+f_{1}+f+f_{2}$ and for $l=4$ and $l=6$ cycles are $f^{\prime}+f_{1}+f_{2}+f$ and $f^{\prime}+f_{1}+\left(C_{4}-f\right)+f_{2}$ respectively, where $C_{k}$ is cycle of length 4

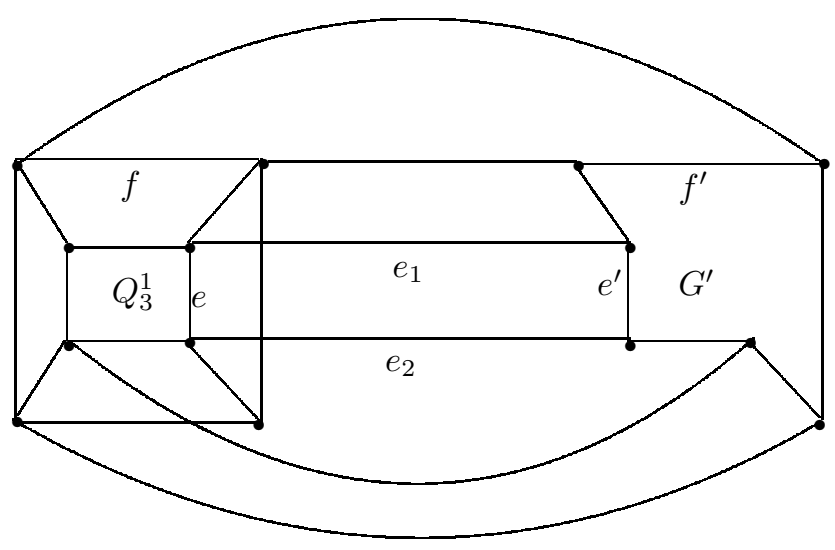

Figure 2

Note: In above lemma if we take $\left|V\left(G^{\prime}\right)\right|=4$ or $\left|V\left(G^{\prime}\right)\right|=8$ we still get the same result by using the almost same technique of proof. 
Lemma 4.4. Consider the subgraphs $\left(Q_{n}, 0\right)$ and $\left(Q_{n}, 1\right)$ of $Q_{n+1}$. Let $G^{\prime}$ be a subgraph of $\left(Q_{n}, 0\right)$ and $R^{\prime}=$ set of cross edges between $\left(Q_{n}, 1\right)$ and $G^{\prime}$. Consider $G=\left(Q_{n}, 1\right) \cup R^{\prime} \bigcup G^{\prime}$. Then $G$ is (a) a bipancyclic graph if $G^{\prime}$ is a Hamiltonian graph for $n \geq 2$.

(b) an edge-bipancyclic graph if $G^{\prime}$ is a bipancyclic graph for $n \geq 3$.

Proof. For edges $e^{\prime}, f_{k}^{\prime} \in E\left(G^{\prime}\right)$ let us denote their corresponding edges in $E\left(Q_{n}, 1\right)$ by $e$ and $f_{k}$ respectively. Also, let us denote $e_{1}, e_{2}, e_{3}, e_{4} \in R^{\prime}$ the edges of which $e_{1}, e_{2}$ join the end vertices of $e$ and $e^{\prime}$, and $e_{3}, e_{4}$ join the end vertices of $f_{k}$ and $f_{k}^{\prime}$.

(a) We want to prove that $G=\left(Q_{n}, 1\right) \bigcup R^{\prime} \bigcup G^{\prime}$ contains a cycle of every even length $l$ for $4 \leq l \leq\left|V\left(Q_{n}\right)\right|+\left|V\left(G^{\prime}\right)\right|$. As $\left(Q_{n}, 1\right)$ is a bipancyclic graph therefore there exists a cycle of every even length $l\left(4 \leq l \leq\left|V\left(Q_{n}\right)\right|\right)$ in $G$.

Consider $C^{\prime}$ a Hamiltonian cycle in $G^{\prime}$ containing an edge say $e^{\prime}$. As $\left(Q_{n}, 1\right)$ is an edge-bipancyclic graph, let $C_{k}$ be a cycle in $\left(Q_{n}, 1\right)$ of even length $k\left(4 \leq k \leq\left|V\left(Q_{n}\right)\right|\right.$ containing $e$. Now we construct a cycle of even length $l=k+\left|V\left(G^{\prime}\right)\right|$ ( therefore $\left|V\left(G^{\prime}\right)\right|+4 \leq l \leq\left|V\left(G^{\prime}\right)\right|+\left|V\left(Q_{n}\right)\right|$ ) as $\left(C^{\prime}-e^{\prime}\right)+e_{1}+\left(C_{k}-e\right)+e_{2}$. For $l=\left|V\left(G^{\prime}\right)\right|+2$ consider $\left(C^{\prime}-e^{\prime}\right)+e_{1}+e+e_{2}$.

(b) For every edge $T \in E(G)$, we want a cycle of every even length $l(4 \leq l \leq|V(G)|)$ passing through $T$.

Case 1: Let $T=e \in E\left(\left(Q_{n}, 1\right)\right)$, as $\left(Q_{n}, 1\right)$ is edge-bipancyclic graph there exists a cycle of even length $l\left(4 \leq l \leq\left|V\left(Q_{n}\right)\right|\right)$ passing through $e$. Now we want cycle of even length $l\left(\left|V\left(Q_{n}\right)\right|+2 \leq\right.$ $\left.l \leq\left|V\left(Q_{n}\right)\right|+\left|V\left(G^{\prime}\right)\right|\right)$ passing through $e$. As $G^{\prime}$ is a bipancyclic subgraph of $\left(Q_{n}, 0\right)$, let $C_{k}^{\prime}$ be a cycle in $G^{\prime}$ of even length $k\left(4 \leq k \leq\left|V\left(G^{\prime}\right)\right|\right)$ passing through an edge say $f_{k}^{\prime}$ (choose $f_{k}^{\prime}$ such that $f_{k}^{\prime} \neq e^{\prime}$ and $f_{k}^{\prime}$ is not adjacent to $\left.e^{\prime}\right)$. By using Lemma 4.1, there always exists Hamiltonian cycle in $\left(Q_{n}, 1\right)$ say $C$ containing $e$ and $f_{k}$. Now we construct a cycle of even length $\left|V\left(Q_{n}\right)\right|+k$ containing $e$ as, $\left(C-f_{k}\right)+e_{3}+\left(C_{k}^{\prime}-f_{k}^{\prime}\right)+e_{4}$, if we take $\left(C-f_{k}\right)+e_{3}+f_{k}^{\prime}+e_{4}$ then we get cycle of length $\left|V\left(Q_{n}\right)\right|+2$ containing $e$.

Case 2: Consider $T=e^{\prime} \in E\left(G^{\prime}\right)$. Let $C_{k}$ denotes a cycle in $\left(Q_{n}, 1\right)$ of even length $k(4 \leq$ $\left.k \leq\left|V\left(Q_{n}\right)\right|\right)$ passing through $e$. Then we construct a cycle of even length $l=k+2$ (therefore $\left.6 \leq l \leq\left|V\left(Q_{n}\right)\right|+2\right)$ in $G$ containing $e^{\prime}$ as $e^{\prime}+e_{1}+\left(C_{k}-e\right)+e_{2}$ and for $l=4$ we take $e^{\prime}+e_{1}+e+e_{2}$. As $G^{\prime}$ is bipancyclic, for every even $k\left(4 \leq k \leq\left|V\left(G^{\prime}\right)\right|-4\right)$ there exists a cycle say $C_{k}^{\prime}$ of length $k$ passing through an edge say $f_{k}^{\prime}$ (we choose $f_{k}^{\prime}$ in such a way that it is not adjacent to an edge $e^{\prime}$ and $\left.f_{k}^{\prime} \neq e^{\prime}\right)$. By using Lemma 4.1, consider a Hamiltonian cycle $C$ in $\left(Q_{n}, 1\right)$ containing $e$ and $f_{k}$. Now a cycle of even length $l=k+\left|V\left(Q_{n}\right)\right|+2$ ( therefore $\left.\left|V\left(Q_{n}\right)\right|+6 \leq l \leq\left|V\left(Q_{n}\right)\right|+\left|V\left(G^{\prime}\right)\right|-2\right)$ containing $e^{\prime}$ in $G$ we construct as $\left((C-e)-f_{k}\right)+e_{1}+e_{2}+\left(\left(C_{k}^{\prime}-e^{\prime}\right)-f_{k}^{\prime}\right)+e_{3}+e_{4}$ and for $l=\left|V\left(Q_{n}\right)\right|+4$, we take $e^{\prime}+e_{1}+\left((C-e)-f_{k}\right)+e_{2}+e_{3}+f_{k}^{\prime}+e_{4}$.

Case 3: And if $T=e_{1}$ where $e_{1} \in R^{\prime}$. Let $e_{1}$ and $e_{2}$ are the edges joining end vertices of corresponding edges $e \in E\left(Q_{n}, 1\right)$ and $e^{\prime} \in E\left(G^{\prime}\right)$. Now to find cycles passing through $e_{1}$ is to same as to find cycles passing through the edge $e^{\prime}$. This completes the proof.

Lemma 4.5. For $n \geq 3$, consider the subgraphs $\left(Q_{n}, 0\right)$ and $\left(Q_{n}, 1\right)$ of $Q_{n+1}$. Let $G_{i}$ be an edgebipancyclic subgraphs of $\left(Q_{n}, i\right)$, for $i \in\{0,1\}$, such that every Hamiltonian cycle in $G_{0}$ contains at least two edges whose corresponding edges are in $G_{1}$ and vice-versa. The set of only those cross edges which join both the end vertices of the corresponding edges from $G_{0}$ and $G_{1}$ be denoted by $R^{\prime}$. Consider $G=G_{0} \bigcup R^{\prime} \cup G_{1}$. Then $G$ is an edge-bipancyclic graph.

Proof. Case 1: Let $e \in E\left(G_{i}\right)$, for $i \in\{0,1\}$. Due to symmetry, it is sufficient to prove that any edge $e \in E\left(G_{1}\right)$ is contained in a cycle of even length $l, 4 \leq l \leq\left|V\left(G_{1}\right)\right|+\left|V\left(G_{0}\right)\right|$. As $G_{1}$ is an edge-bipancyclic graph, therefore, $e$ is contained in a cycle of even length $l, 4 \leq l \leq\left|V\left(G_{1}\right)\right|$.

Let $C$ denotes Hamiltonian cycle in $G_{1}$ containing $e$. By assumption, there exists at least one edge of $C$ other than $e$ say $f$ such that its corresponding edge $f^{\prime}$ is in $G_{0}$. Thus if $f=\left\langle A_{1}, A_{2}\right\rangle$ then 
$e_{1}=\left\langle A_{1}, B_{1}\right\rangle$ and $e_{2}=\left\langle A_{2}, B_{2}\right\rangle$ such that $e_{1}, e_{2} \in R^{\prime}$ where $A_{1}, A_{2} \in V\left(G_{1}\right)$ and $B_{1}, B_{2} \in V\left(G_{0}\right)$. Now let us denote by $C_{k}^{\prime}$ a cycle of even length $k\left(4 \leq k \leq\left|V\left(G_{0}\right)\right|\right)$ containing $f^{\prime}$ in $G_{0}$, Now we construct cycle of even length $l=k+\left|V\left(G_{1}\right)\right|$ (as $4 \leq k \leq\left|V\left(G_{0}\right)\right|$, therefore $|C|+4 \leq l \leq$ $\left.\left|V\left(G_{1}\right)\right|+\left|V\left(G_{0}\right)\right|\right)$ containing $e$ as $(C-f)+e_{1}+\left(C_{k}^{\prime}-f^{\prime}\right)+e_{2}$ and for $l=|C|+2$ we take cycle $(C-f)+e_{1}+f^{\prime}+e_{2}$.

Case 2: For $e_{1} \in R^{\prime}$. By assumption there exists $e_{2} \in R^{\prime}$, such that $e_{1}$ and $e_{2}$ are the edges joining end vertices of corresponding edges $f \in E\left(G_{1}\right)$ and $f^{\prime} \in E\left(G_{0}\right)$. Let $C_{k}$ denotes a cycle of even length $k\left(4 \leq k \leq\left|V\left(G_{1}\right)\right|\right)$ containing $f$ in $G_{1}$. Now we construct a cycle of even length $l=k+2$ ( therefore $\left.6 \leq l \leq\left|V\left(G_{1}\right)\right|+2\right)$ containing $e_{1}$ as $\left(C_{k}-f\right)+e_{1}+f^{\prime}+e_{2}$ and for $l=4$ we take $f+e_{1}+f^{\prime}+e_{2}$. Let $C$ be the Hamiltonian cycle containing an edge $f$ in $G_{1}$ and let $C_{k}^{\prime}$ be denotes cycle of length $k\left(4 \leq k \leq\left|V\left(G_{0}\right)\right|\right.$ containing $f^{\prime}$ in $G_{0}$. Now we construct cycle of even length $l=\left|V\left(G_{1}\right)\right|+k$ ( therefore $\left.\left|V\left(G_{1}\right)\right|+4 \leq l \leq\left|V\left(G_{1}\right)\right|+\left|V\left(G_{0}\right)\right|\right)$ containing $e_{1}$ as $(C-f)+e_{1}+\left(C_{k}^{\prime}-f^{\prime}\right)+e_{2}$.

Lemma 4.6. For $n \geq 3$, consider the subgraphs $\left(Q_{n}, 0\right)$ and $\left(Q_{n}, 1\right)$ of $Q_{n+1}$. Let $G_{i}$ be the subgraph of $\left(Q_{n}, i\right)$, for $i \in\{0,1\}$. If an even length cycle $C$ in $Q_{n+1}$ contains at least 3 vertices of $G_{i}$ for all $i \in\{0,1\}$, then $|E(C)| \cap\left|E\left(G_{i}\right)\right| \geq 2$ for all $i \in\{0,1\}$.

Proof. For every vertex in $\left(Q_{n}, 0\right)$ there exists a unique vertex in $\left(Q_{n}, 1\right)$ which is adjacent to it, hence the proof follows immediately.

Lemma 4.7. For $n \geq 3$, let $F$ denotes a strongly independent set and $P$ denotes a spanning subgraph of $Q_{n}$ such that for every vertex $v$ of $Q_{n}, d_{p}(v)=1$ or 2 . Then $\left|E\left(Q_{n}-F\right)\right| \cap|E(P)| \geq 2$.

Proof. As $P$ is a spanning subgraph, every vertex $u \in V(F)$ lies on $P$. If $|F|=1$, then we are through. Now let $|F| \geq 2$. Let $u, v \in F$, and let us denote by $u_{i}$ and $v_{i}$ the $n$ neighbors of $u$ and $v$ in $Q_{n}$ respectively $(1 \leq i \leq n)$. Let $u u_{1}, u u_{2} \in E(P)$. Due to trianglefreeness of $Q_{n}, u_{i}(3 \leq i \leq n)$ can not be adjacent to $u_{1}$ and $u_{2}$, but $d_{p}\left(u_{i}\right)=1$ or 2 , therefore, $u_{i}(3 \leq i \leq n)$ is adjacent to some vertex $w_{i}$ in $P$. We note that $w_{i} \notin F$ otherwise it will contradict strongly independentness of $F$. By similar arguments, $v_{i}(3 \leq i \leq n)$ is adjacent to some vertex $w_{i}^{\prime}$ in $P$ which is not in $F$. Thus, we proved.

For any $x \in Q_{n}$ the mapping $u \longrightarrow u+x$ is a graph automorphism of $Q_{n}$ also for any permutation $\sigma \in S_{n}$, the mapping $\left(v_{1}, v_{2}, \ldots, v_{n}\right) \longrightarrow\left(v_{\sigma 1}, v_{\sigma 2}, v_{\sigma 3}, \ldots ., v_{\sigma n}\right)$ is also a graph automorphism of $Q_{n}$, therefore the following result is true in view of Lemma 3.1

Lemma 4.8. If $D$ is a PID set of $Q_{n}$ such that $Q_{n}-D$ is edge-bipancyclic then;

(a) $Q_{n}-D_{i}$ is also edge-bipancyclic where for $1 \leq i \leq n, D_{i}=D+e_{i}$ and $e_{i}=(0,0, \ldots, 1,0 \ldots, 0)$ (1 is at $i^{\text {th }}$ position);

(b) in addition, if $D$ is linear then for any linear perfect independent dominating set $D_{0}$ of $Q_{n}$, $Q_{n}-D_{0}$ is also edge-bipancyclic.

Now we prove our main result of this section which gives edge-bipancyclicity of a hamming shell.

Theorem 4.9. For any coset of any linear PID set $D$ of $Q_{n}\left(n=2^{m}-1\right.$ and $\left.m \geq 3\right), Q_{n}-D$ is edge-bipancyclic.

Proof. We follow notations of Observation 3.2 .

Part I : We decompose $Q_{7}$ as $Q_{7}=Q_{3} \times Q_{4}$. Let $C=t_{1} t_{2} t_{3} \ldots . . t_{16} t_{1}$ be a Hamiltonian cycle in $Q_{4}$. Without loss of generality say $t_{j}$ is of odd parity, for odd integers $j, 1 \leq j \leq 16$. Obviously, $t_{j}$ is of even parity, for even integers $j, 1 \leq j \leq 16$ (see Figure 3). 


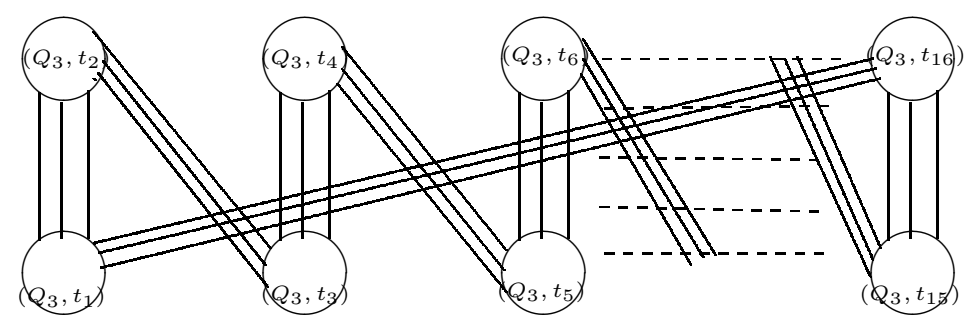

Figure 3

Let $D_{0}^{\prime}$ be the Kernel of matrix $H^{\prime}$ and let $D_{i}^{\prime}=D_{0}^{\prime}+e_{i}$ (for $1 \leq i \leq 3, e_{i}=(0, . .1, . .0) 1$ is at $i^{t h}$ position) be its cosets. Now by lemma 3.1 and observation 3.2 , we get the kernel of $H$ say $D$ such that for odd integer $j, D \cap\left(Q_{3}, t_{j}\right)=\phi$ and for even integer $j, D \cap\left(Q_{3}, t_{j}\right)=\left(D^{\prime}, t_{j}\right)$ where $D^{\prime} \in\left\{D_{0}^{\prime}, \ldots, D_{3}^{\prime}\right\}(1 \leq j \leq 16)$.

Now we claim that $Q_{7}-D$ is edge-bipancyclic.

Let us denote by $G_{j}=\left(Q_{3}, t_{j}\right)-\left(D \cap\left(Q_{3}, t_{j}\right)\right)(1 \leq j \leq 16)$. For every even integer $j(1 \leq j \leq 16)$, $G_{j}$ is isomorphic to one of the $G_{i}^{\prime}\left(G_{i}^{\prime}=Q_{3}-D_{i}^{\prime}\right.$, see Lemma 4.2) which is a Hamiltonian cycle $(0 \leq i \leq 3)$. And for odd integer $j, G_{j}=\left(Q_{3}, t_{j}\right)$ which is isomorphic to $Q_{3}$.

Let us denote by $E_{j}$ the set of cross edges between $G_{j}$ and $G_{j+1}(1 \leq j \leq 15)$, therefore $E_{j}=\{e$ : one end vertex of an edge $e$ is in $G_{l}, l$ is an even integer with $\left.l \in\{j, j+1\}\right\}$ (see Figure 4).

Let $H_{j}=G_{j} \bigcup E_{j} \bigcup G_{j+1}$ ( $j$ is an odd integer, $1 \leq j \leq 16$ ), by Lemma 4.3. $H_{j}$ is an edge bipancyclic graph (see Figure 5).

As $V\left(Q_{7}-D\right)=\bigcup_{j=1}^{16} V\left(G_{j}\right)$. (Also, it is well known that $Q_{n}$ for any $n \geq 1$ has a Hamiltonian path between every two vertices of opposite parity [6] so we can take some other Hamiltonian cycle in $Q_{4}$ instead of cycle $t_{1} t_{2} \ldots t_{16} t_{1}$ but due to bipartiteness of hypercubes the cross edges $E_{i}$ will in between $G_{j}$ (for $j$ odd only) and $G_{k}$ (for $k$ even only) for some $1 \leq j, k \leq 16$.) Hence, it is sufficient to prove that $H_{1} \bigcup E_{2} \bigcup H_{3}$ is edge-bipancyclic .

By using Lemma 4.6. we observe that every Hamiltonian cycle in $H_{1}$ contains at least two edges of $G_{2}$. And by using Lemma 4.7 we observe that every Hamiltonian cycle $C$ in $H_{3}$ contains at least two edges of $G_{3}$ which corresponds to the edges of $G_{2}$.

Now by using Lemma 4.5, we observe that $H_{1} \bigcup E_{2} \bigcup H_{3}$ is edge-bipancyclic.

Thus we proved the existence of linear perfect independent dominating set of $Q_{7}$ such that $Q_{7}-D$ is edge-bipancyclic.

By Lemma 4.8 it is sufficient what we proved that there exists a linear perfect independent dominating set $D$ of $Q_{7}$ such that $Q_{7}-D$ is edge-bipancyclic. 


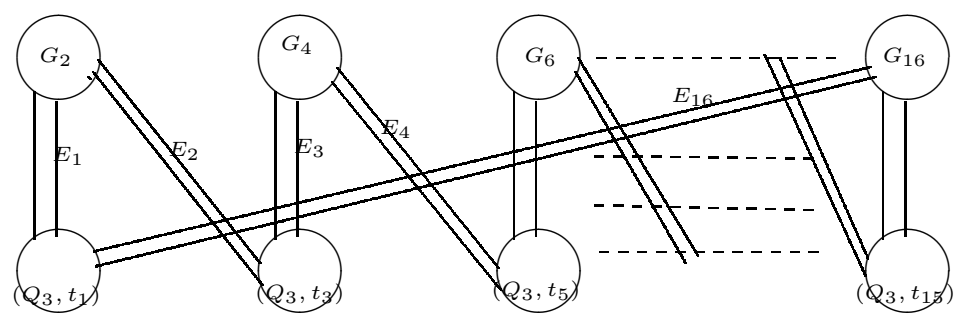

Figure 4

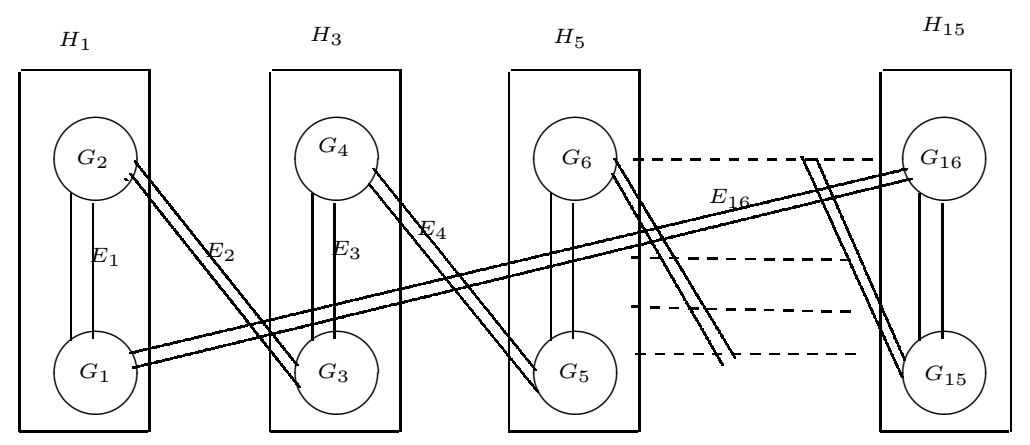

Figure 5

Part II : Now we prove the result by induction on $m . Q_{7}-D^{\prime}$ is edge-bipancyclic shows our statement is true for $m=3$ (that is $n=2^{3}-1=7$ ). Suppose the statement is true for $m=r \geq 3$. Let $n=2^{r}-1$, therefore, $Q_{n}-D^{\prime}$ is edge-bipancyclic where $D^{\prime}$ denotes any linear PID set or its coset in $Q_{n}$.

We want to show that the statement is true for $m=r+1$. Let $k=2^{r+1}-1=n+(n+1)$.

We decompose $Q_{k}$ as $Q_{k}=Q_{n} \times Q_{n+1}$. Let $C=t_{1} t_{2} t_{3} \ldots t_{2^{n+1}} t_{1}$ be a Hamiltonian cycle in $Q_{n+1}$. Without loss of generality say $t_{j}$ is of odd parity, for odd integer $j, 1 \leq j \leq 2^{n+1}$. Obviously, $t_{j}$ is of even parity, for even integer $j, 1 \leq j \leq 2^{n+1}$.

Let $D_{0}^{\prime}$ be the Kernel of matrix $H^{\prime}$ and let $D_{i}^{\prime}=D_{0}^{\prime}+e_{i}\left(\right.$ for $1 \leq i \leq n, e_{i}=(0, \ldots, 1, \ldots, 0) 1$ is at the $i^{t h}$ position) be its cosets. Now by lemma 3.1 and observation 3.2, we get the kernel of $H$ say $D$ such that for odd integer $j, D \cap\left(Q_{n}, t_{j}\right)=\phi$ and for even integer $j, D \cap\left(Q_{n}, t_{j}\right)=\left(D^{\prime}, t_{j}\right)$ where $D^{\prime} \in\left\{D_{0}^{\prime}, \ldots, D_{n}^{\prime}\right\}\left(1 \leq j \leq 2^{n+1}\right)$.

We claim that $Q_{k}-D$ is edge-bipancyclic.

Let us denote by $G_{j}=\left(Q_{n}, t_{j}\right)-\left(D \cap\left(Q_{n}, t_{j}\right)\right)\left(1 \leq j \leq 2^{n+1}\right)$. For every even integer $j$ $\left(1 \leq j \leq 2^{n+1}\right), G_{j}$ is isomorphic to one of the $G_{i}^{\prime}\left(G_{i}^{\prime}=Q_{n}-D_{i}^{\prime}\right)$ and by induction $G_{i}^{\prime}$ is an 
edge-bipancyclic graph $(0 \leq i \leq n)$. For odd integer $j\left(1 \leq j \leq 2^{n+1}\right), G_{j}=\left(Q_{n}, t_{j}\right)$ is isomorphic to $Q_{n}$.

Let us denote by $E_{j}$ the set of cross edges between $G_{j}$ and $G_{j+1}\left(1 \leq j \leq 2^{n+1}-1\right)$, therefore $E_{j}=\left\{e:\right.$ one end vertex of an edge $e$ is in $G_{l}, l$ is an even integer with $\left.l \in\{j, j+1\}\right\}$.

By $(b)$ part of Lemma 4.4, we get $H_{j}=G_{j} \bigcup E_{j} \bigcup G_{j+1}\left(j\right.$ is an odd integer, $\left.1 \leq j \leq 2^{n+1}\right)$, is an edge bipancyclic graph.

As $V\left(Q_{k}-D\right)=\bigcup_{j=1}^{2^{n+1}} V\left(G_{j}\right)$. It is sufficient to prove that $H_{1} \bigcup E_{2} \bigcup H_{3}$ is edge-bipancyclic.

By using Lemma 4.6, we observe that every Hamiltonian cycle in $H_{1}$ contains at least two edges of $G_{2}$. And by using Lemma 4.7 we observe that every Hamiltonian cycle $C$ in $H_{3}$ contains at least two edges of $G_{3}$ which corresponds to the edges of $G_{2}$.

Now by using Lemma 4.5. we observe that $H_{1} \bigcup E_{2} \bigcup H_{3}$ is edge-bipancyclic.

Thus we proved that $Q_{k}-D$ is edge-bipancyclic for any linear perfect independent dominating set or its coset $D$ in $Q_{k}$.

\section{Connectivity with Distant Faulty Vertices}

In this section, we study the connectivity of hypercubes by removing a restricted set of faulty vertices say $F$ such that each pair of faulty vertices is distant. Here 'distant' or 'strongly independent' means that the distance between two vertices $u$ and $v$ is greater than or equal to $3, d(u, v) \geq 3$. Clearly, each non-faulty vertex is adjacent to at most one faulty vertex. It is proved that (see[6]) the set $F$ can have up to $2^{n-1}$ vertices in $Q_{n}$.

Here, we prove that for strongly independent set $F$ of $Q_{n}, Q_{n}-F$ is $(n-1)$-connected for $n \geq 3$.

It is important to see that, which vertex faulty hypercubes maintain the property like connectivity. In the following theorem, we prove the existence of such vertex faulty hypercubes.

Theorem 5.1. For $n \geq 3$, let $F$ be a strongly independent set in $Q_{n}$ then $Q_{n}-F$ is $(n-1)$-connected.

Proof. We prove the theorem by induction on $n$.

In $Q_{3}$, a strongly independent set $F$ is a single vertex in $Q_{3}$ or $F=\{(000),(111)\}$ or $F=$ $\{(100),(011)\}$ or $F=\{(010),(101)\}$ or $F=\{(001),(110)\}$. We can easily observe that $Q_{3}-F$ is 2 -connected graph, see Figure 1. Thus result holds for $n=3$.

Suppose $n \geq 4$. We assume the result for $n-1$. Consider the subgraphs $\left(Q_{n-1}, 0\right)$ and $\left(Q_{n-1}, 1\right)$ of $Q_{n}$ and the set $R$ of cross edges between them. So, $Q_{n}=\left(Q_{n-1}, 0\right) \cup\left(Q_{n-1}, 1\right) \cup R$. Let $F$ be a strongly independent set of $Q_{n}$ and let $G=Q_{n}-F$. Let us denote by $F_{0}$ and $F_{1}$ the strongly independent sets $V\left(Q_{n-1}, 0\right) \bigcap F$ and $V\left(Q_{n-1}, 1\right) \cap F$ respectively. It is easy to observe that $G=G_{0} \cup G_{1} \cup R^{\prime}$ where $G_{0}=\left(Q_{n-1}, 0\right)-F_{0}, G_{1}=\left(Q_{n-1}, 1\right)-F_{1}$ and $R^{\prime} \subset R$ such that $R^{\prime}=\left\{\langle u, v\rangle=u v: u \in V\left(G_{0}\right)\right.$ and $\left.v \in V\left(G_{1}\right)\right\}$ Without loss let $\left|V\left(G_{0}\right)\right|=k \leq\left|V\left(G_{1}\right)\right|=t$ (observe that $\left.2^{n-2} \leq k \leq t\right)$, we can label the vertices of $G_{0}$ using the set $\left\{u_{1}, u_{2}, u_{3}, \ldots . ., u_{k}\right\}$ and $G_{1}$ using the set $\left\{v_{1}, v_{2}, v_{3}, \ldots . ., v_{t}\right\}$, in such a way that $R^{\prime}=u_{1} v_{1}, u_{2} v_{2}, u_{3} v_{3}, \ldots . u_{m} v_{m}$, where $2^{n-2} \leq m \leq k$. By induction hypothesis, $G_{0}$ and $G_{1}$ are $(n-2)$-connected.

Claim : $G=Q_{n}-F$ is $(n-1)$-connected.

Let $S \subset V(G)$ with $|S|=n-2$. It suffices to prove that $G-S$ is connected.

There are at least $2^{n-2}-|S|=2^{n-2}-(n-2) \geq 1$ edges from the set $R^{\prime}$ which join $G_{0}$ to $G_{1}$ in $G-S$ Suppose that $S \subset V\left(G_{0}\right)$ or $S \subset V\left(G_{1}\right)$. Without loss, we assume $S \subset V\left(G_{0}\right)$. If $G_{0}-S$ is connected or each component of $G_{0}-S$ has a neighbor in $G_{1}$, then $G-S$ is connected because $G_{1}$ is connected. Assume that $G_{0}-S=\left(Q_{n-1}, 0\right)-F_{0}-S$ is disconnected and has a component $W$ having no neighbor in $G_{1}$. As $\left(Q_{n-1}, 0\right)$ is $(n-1)$-connected, $\left(Q_{n-1}, 0\right)-S$ is connected. Therefore, $W$ contains at least one vertex say $u_{i}$ which is adjacent to vertex from $F_{0}$ only, say $u_{\sigma(i)}$. As $u_{\sigma(i)} \in F_{0}$ therefore corresponding vertex of $u_{i}$ from $\left(Q_{n-1}, 1\right)$ say $v_{i} \notin F_{1}$ so $v_{i} \in V\left(G_{1}\right)$. Hence, $W$ is joined to the connected graph $G_{1}$ by an edge $\left\langle u_{i}, v_{i}\right\rangle \in R^{\prime}$. 
Suppose, instead that $S$ intersect both $V\left(G_{0}\right)$ and $V\left(G_{1}\right)$. Let $S_{0}=V\left(G_{0}\right) \cap S$ and $S_{1}=V\left(G_{1}\right) \cap S$. Then $S=S_{0} \cup S_{1}$ and $S_{0} \cap S_{1} \neq \phi$. Since $|S| \leq n-2,\left|S_{0}\right| \leq n-3$ and $\left|S_{1}\right| \leq n-3$. Hence, both $G_{0}-S_{0}$ and $G_{1}-S_{1}$ are connected because $G_{0}$ and $G_{1}$ are $(n-2)$-connected. Further $G_{0}-S_{0}$ and $G_{1}-S_{1}$ are joined to each other by at least one edge of $R^{\prime}$ in $G-S$. Therefore, $G-S$ is connected. Thus $G$ is $(n-1)$-connected.

\section{Connectivity and Bipancyclicity with Faulty Vertices in Dominating Set.}

We are interested in perfect dominating sets because their removal results in a subgraph in which each vertex has degree one less than in the original graph. In this section, we prove the existence of perfect dominating set $D$ of a hypercube $Q_{n}(n \geq 3)$ such that $Q_{n}-D$ is $(n-1)$-regular, $(n-1)$ connected and bipancyclic.

By using Theorem 4.9 and Theorem [5.1, we obtain the following Theorem which states that the Hamming shell is regular, connected and edge-bipancyclic.

Theorem 6.1. For $n=2^{k}-1, k \geq 2$, let $D$ denote a perfect independent dominating set in $Q_{n}$. Then $Q_{n}-D$ is $(n-1)$-regular and $(n-1)$-connected and in addition, if $D$ is any coset of any linear perfect independent dominating set of $Q_{n}$ then $Q_{n}-D$ Hamiltonian for $k=2$ and edge-bipancyclic for $k \geq 3$. Moreover, this is the smallest set satisfying the above property.

Proof. A perfect independent dominating set $D$ in $Q_{n}$ for $n=2^{k}-1, k \geq 2$ is a strongly independent set in $Q_{n}$ and hence $Q_{n}-D$ is $(n-1)$-regular where $|D|=2^{n-k}$. Also by Theorem 5.1, $Q_{n}-D$ is $(n-1)$-connected.

If $D$ is any coset of any linear perfect independent dominating set of $Q_{n}$ then $Q_{n}-D$ is Hamiltonian for $k=2$ and edge-bipancyclic for $k \geq 3$ (by Theorem 4.9). Thus we proved the existence of perfect independent dominating set $D$ of $Q_{n}$ such that $Q_{n}-D$ is $(n-1)$-regular, $(n-1)$-connected and Hamiltonian for $k=2$ and edge-bipancyclic for $k \geq 3$.

In case if we choose a set of vertices in $Q_{n}$ say $F_{v}$ such that $\left|F_{v}\right|=2^{n-k}-1$ then because of $n$-regularity of $Q_{n}$ exactly $(n+1)$ vertices remain unattended which shows $Q_{n}-F_{v}$ is non-regular, therefore $D$ is the smallest set satisfying the property above.

We need following lemmas before proving the main Theorem of this section.

Lemma 6.2 ([1]). If $G$ is an n-regular graph, then a perfect dominating set of $G$ can be described as follows: If $H$ is an $(n-1)$-regular induced subgraph of $G$, then $V(G)-V(H)$ is a perfect dominating set of $G$.

Lemma 6.3 ([9]). Let for $k \geq 2, P_{k}$ be denoted the path of length $k-1$. Then the mesh $P_{m} \times P_{n}$ $(m, n \geq 2)$ contains a cycle of length $l$ for any even integer $l$ with $4 \leq l \leq m n$.

Lemma $6.4([10])$. For any connected graphs, $G$ and $H, \kappa(G \times H) \geq \kappa(G)+\kappa(H)(\kappa(G)$ denotes connectivity of $G$ ).

Now we prove existence of a total perfect dominating set of hypercubes removal of which gives a regular, connected and bipancyclic subgraph.

Theorem 6.5. In an $n$-dimensional hypercube $Q_{n}(n \geq 4)$ there exists a total perfect dominating set $D$ of $Q_{n}$ for $2^{k}-1<n<2^{k+1}-1$ and $k \geq 2$ with $|D|=2^{(n-k)}$ such that $Q_{n}-D$ is $(n-1)$-regular, $(n-1)-$ connected and bipancyclic.

Proof. For $n \geq 4$ consider $k \geq 2$ such that $2^{k}-1<n<2^{k+1}-1$. Now we write $Q_{n}=Q_{m} \times Q_{n-m}$ where $m=2^{k}-1$. For any $t \in V\left(Q_{n-m}\right)$ we denote by $\left(Q_{m}, t\right)$ the subgraph of $Q_{n}$ induced by the vertices whose last $(n-m)$ components form the tuple $t$.

By Theorem 4.9, for every $D$ which is any coset of any linear perfect independent dominating set of $Q_{m}, H=Q_{m}-D$ is $(m-1)$-regular, $(m-1)$-connected and, in addition, it is Hamiltonian for 
$k=2$, and edge-bipancyclic for $k \geq 3$.

Claim : The subgraph $G=H \times Q_{n-m}$ of $Q_{n}$ is $(n-1)$-regular, $(n-1)$-connected and bipancyclic. For $s \in V(H)$ and $t \in V\left(Q_{n-m}\right)$ we denote by $(s, t)$ the vertex in $Q_{n}$ whose first $m$ components form the tuple $s$ and whose last $(n-m)$ components form the tuple $t$. Thus we have $V(G)=$ $V(H) \times V\left(Q_{n-m}\right)=\left\{(s, t): s \in V(H), t \in V\left(Q_{n-m}\right)\right\}$.

Firstly, we prove that $G$ is $(n-1)$-regular.

As degree of $s$ that is $d(s)=m-1$ in $H$ and $d(t)=n-m$ in $Q_{n-m}$, we have $d(s, t)=(m-1)+$ $(n-m)=n-1$ in $G$, for every vertex in $G$.

Now, we will prove that $G$ is $(n-1)$-connected.

We know that $\kappa(G) \leq \delta(G)=n-1$. Also by Lemma 6.4 $\kappa(G)=\kappa\left(H \times Q_{n-m}\right) \geq \kappa(H)+\kappa\left(Q_{n-m}\right)=$ $(m-1)+(n-m)$ gives us $\kappa(G)=n-1$.

Lastly, we will prove that $G$ is bipancyclic for $k \geq 3$.

As $G=H \times Q_{n-m}$ contains the mesh $P_{m\left(2^{m-k}\right)} \times P_{2^{n-m}}$ as a spanning subgraph, bipancyclicity of $G$ follows by the Lemma 6.3

For $t \in V\left(Q_{n-m}\right)$, let us denote by $D^{\prime}=\bigcup(D, t)$ where $V(D, t)=\left\{(s, t): s \in V(D), t \in V\left(Q_{n-m}\right)\right\}$. We note that $G=Q_{n}-D^{\prime}$.

By Lemma 6.2, $D^{\prime}$ is a perfect dominating set of $Q_{n}$ and in addition, $D^{\prime}$ is total by definition. Thus $D^{\prime}$ is a total perfect dominating set of $Q_{n}$ removal of which gives an induced subgraph $G$ which is $(n-1)$-regular, $(n-1)$-connected and bipancyclic.

This completes the proof.

\section{Concluding remarks}

Thus we proved that for any coset of any linear perfect independent dominating set $D$ of $Q_{n}, Q_{n}-D$ is edge-bipancyclic. Whether the same property is true for any perfect independent dominating set of $Q_{n}$ ?

Acknowledgment: The author gratefully acknowledges the Department of Science and Technology, New Delhi, India for the award of Women Scientist Scheme (SR/WOS-A/PM-79/2016) for research in Basic/Applied Sciences.

$* * * * * * * * * * * *$

\section{REFERENCES}

1. J. Borges, I. J. Dejter, On perfect dominating sets in hypercubes and their complements, J. Combin. Math. Combin. Comput., 20(1996), $161-173$.

2. I. J. Dejter, On symmetric subgraphs of the 7-cube: an overview, Discrete Math., 124(1994), 55 - 66 .

3. I. J. Dejter, Symmetry of factors of the 7-cube Hamming shell, J. Combin. Des., 5(1997), $301-309$.

4. I. J. Dejter, Equitable factorizations of Hamming shells, Discrete Math., 261(2003), 177 - 187.

5. W. Duckworth, P. E. Dunne, A. M. Gibbons, M. Zito, Leafy spanning Trees in Hypercubes, Appl. Math. Lett., 14(2001), $801-804$

6. Petr Gregor, Riste Skrekovski, Long cycles in hypercubes with distant faulty vertices, Discrete Math. Theoret. Comput. Science, 11(1)(2009), $187-200$.

7. D. G. Hoffman, D. A. Leonard, C. C. Lindner, K. T. Phelps, C. A. Rodger, J. R. Wall, Coding Theory, The Essentials, M. Dekker Inc, New York, 1991.

8. San Ling, Chaoping Xing, Coding Theory A First Course, First Edition, Cambridge University Press, New York, 2004.

9. S. A. Mane, B. N. Waphare, Regular connected bipancyclic spanning subgraphs of hypercubes, Comput. Math. Appl., 62(2011), $3551-3554$.

10. S. Spacapan, Connectivity of cartesian products of graphs, Appl. Math. Lett., 21(2008), $682-685$.

11. P. M. Weichsel, Dominating sets of $n$-cubes, J. Graph Theory, 18(1994), $479-488$.

12. Douglas. B. West, Introduction to Graph theory, Second Edition, Prentice-Hall of India, New Delhi, 2002.

13. J. M. Xu, Z. Z. Du, M. Xu, Edge fault tolerant edge bipancyclicity of hypercubes, Inform. Process. Lett., 96(2005), $146-150$. 